\title{
Cancer Patients Treatment Management during COVID-19 Pandemic: A Single Center Experience at Bharatpur, Nepal
}

\section{Yadav Ajay Kumar',2*, Gnawali Suman², Chapagain Dipak ${ }^{3}$, Yadav Dipesh Kumar $^{4}$ and Gangbiao Yuan ${ }^{1}$}

${ }^{1}$ Department of Nuclear Medicine, The Second Affiliated Hospital of Chongqing

Medical University, China

${ }^{2}$ Department of Radio-Diagnosis, Imaging and Nuclear Medicine, BP Koirala

Memorial Cancer Hospital, Bharatpur, Chitwan, Nepal

${ }^{3}$ Department of Pathology, BP Koirala Memorial Cancer Hospital, Bharatpur,

Chitwan, Nepal

${ }^{4}$ Department of Medical Record, BP Koirala Memorial Cancer Hospital, Bharatpur,

Chitwan, Nepal

*Corresponding Author: Yadav Ajay Kumar, Department of Nuclear Medicine, The

Second Affiliated Hospital of Chongqing Medical University, China.
Received: June 01, 2021

Published: July 13, 2021

(C) All rights are reserved by Yadav Ajay

Kumar., et al.

\section{Abstract}

Since last December 2019, there is panic situation all over the world due to COVID-19 pandemic. Due to highly infectious nature of SARS-CoV-2, all other non-communicable diseases treatment is directly affected in most of the hospitals. Most hospitals in epidemic region reduced the non-emergency treatments and other supportive services. Our country Nepal is also vigorously infected by COVID-19. Before recovering from first wave of that epidemic, there is second wave ofSARS-CoV-2 with double and triple mutant virus which one seems more dangerous than the previous. We are still facing the more panic situation of this epidemic. As a result, it is difficult to manage treatment of cancer patients in hospital in time, which adversely affects the prognosis. To overcome from that issue, our cancer center develops a strategic plan to continue the treatment of patients and at the same time prevention is also important for medical staffs, non-infected patients and visitors. In our study, we included that how our cancer center made a plan to continue treatment of cancer patients, preventive major to hospital staffs and preventive major to visitors with patients. We concluded that adding RT-PCR screening facility and separate COVID isolation ward including all preventive majors regarding COVID-19 to our hospital, makes more impact on cancer patients treatment management during COVID-19 pandemic.

Keywords: COVID-19; Cancer; SARS-CoV-2; RT-PCR

\section{Introduction and Background}

First identified case of COVID-19 (named by WHO of Corona Virus Disease 2019) was found in Wuhan China which is an infectious disease caused by severe acute respiratory syndrome corona virus (SARS-CoV-2). Worldwide total diagnosed case of COVID-19 was $159,732,343$ patients, global total death due to this disease was reported 3,319,169 and total 95,819,194 patients were recovered from COVID-19 till date May 12, 2021 [1]. It is increasing day by day worldwide. Some of the countries maintain the disciplined life style according to COVID-19 protocol and it is seems that there is control of infection rate and death rate due to COVID-19 in those countries. Some of the countries are still worsely affected and both the infection rate and death rate are too high. 
The most common symptoms of COVID-19 are Fever, Dry cough, Fatigue and other symptoms that are less common and may affect some patients include loss of taste or smell, nasal, congestion, conjunctivitis, sore throat, headache, muscle or joint pain, different types of skin, rash, nausea or vomiting, diarrhea, chills or dizziness [2]. Symptoms of severe COVID-19 disease include shortness of breath, loss of appetite, confusion, persistent pain or pressure in the chest, high temperature (above $38^{\circ} \mathrm{C}$ ) and other less common symptoms are irritability, reduced consciousness (sometimes associated with seizures), anxiety, depression, sleep disorders, more severe and rare neurological complications such as strokes, brain inflammation, delirium and nerve damage [2]. These are not only all the possible symptoms. CDC will continue to update this list as we learn more about COVID-19. Older adults and people who have severe underlying medical conditions like heart or lung disease or diabetes seem to be at higher risk for developing more serious complications from COVID-19 illness [3]. It is concluded by many literatures that there are majority of asymptomatic COVID-19 patients. One literature concluded that about $15.6 \%$ of confirmed COVID-19 patients are asymptomatic [4]. It was also reported that nearly $50 \%$ of the patients with no symptoms at detection time will develop symptoms later and asymptomatic COVID-19 patients could have laboratory in accuracy and radiational manifestations which can be used as screening strategies to identify asymptomatic infection [4].

National government data showed that our country Nepal is also badly hit by COVID-19. According to data updated by government of Nepal, total diagnosed case of COVID-19 was 422349 patients, total death due to this disease was reported 4252 and total 316463 patient were recovered from COVID-19 in Nepal till date May 12, 2021 [5].

Post chemotherapy symptoms of cancer patients are headache, diarrhea, nausea, and vomiting which are very likely to COVID-19 patient symptoms [6]. Cancer patients are highly prone to viral as well as bacterial pneumonia because chemotherapy and radiotherapy treatment of cancer patients effect on immunity and reduce the immunity of human body [7]. Due to this, there is difficult to distinguish COVID-19 infected patients from non-infected patients during epidemic of COVID-19 for cancer patients. This is the main reason, most of the cancer center facing problem to run full phase treatment procedure and reduced the facility only for emergency patients. On the other hand, early treatment of cancer patients increase the prognosis and delaying in treatment decrease the probability of prognosis. During COVID-19 epidemic, it was difficult to manage treatment of cancer patients in hospital in time, which adversely affects the prognosis and may sometimes lead to severe consequences. There are several guidelines were announced by Nepal Government regarding treatment of patients with other diseases than COVID-19. Initially, it was seems that it will controlled in few times but till now it is out of control in our country Nepal. During previous few weeks, our center (B.P. Koirala Memorial Cancer Hospital Bharatpur, Nepal) totally stopped OPD services except emergency services. When it was insured that this epidemic COVID-19 will not controlled in Nepal within few weeks then our center started working on protocol to open OPD and other services with all precautions regarding COVID-19 and how to handle preventive major to hospital staffs, cancer patients and visitors with patients.

Taking into account the potential of epidemic COVID-19, our hospital made a committee of few experts members to develop a strategy to train the all hospital staffs for safety majors regarding SARS-CoV-2 and a set of infection-control interventions and develop a setup for screening of the nucleic acid of SARS-CoV-2. After that our center stringently followed the interventional protocols with and safety from April 2021 to till now.

The actual risk of COVID-19 to cancer patients is still unclear. There are several literatures reported that COVID-19 make high risk to cancer patients. One of the study concluded that carcinoma patients and SARS-CoV-2 are more probable to worsen into severe sickness than those without carcinoma [8]. One research paper reported that 12 among 18 cancer patients which were infected with SARS-CoV-2 virus, there is no signs of a weakened immune response [9]. It is also concluded that infected patients didn't well represent the immune status of the majority of patients in recovery from cancer treatment [9].

Cancer patients are caught on the crossfire of this pandemic as a unique group with increased risks of contracting COVID-19. Their weakened immunity is resulting from a myriad of factors including underlying malignancy burden and active cancer treatments like cytotoxic chemotherapy, radiotherapy, or even due to patients undergoing transplant and use of immune suppressants to avoid rejection.

Moreover, distraction effects that involve diverting attention to the pandemic as compared to cancer patients cause collateral loss and unfavorable outcomes in oncology care. Instances, where even oncology care clinicians are in the frontlines to care for COVID -19 patients, are reported in many institutions due to shortages of staff. 
In the middle- and low-income countries with the ailing healthcare system and sub-optimal oncology care, these distraction effects are far worse. The inability to deliver palliative care or primary palliative care to patients is another significant concern due to forced quarantine. There could be a double wave of cancer patients' deaths: a surge in immune-compromised patients who contract COVID-19 or those whose treatments were de-escalated, canceled, or delayed.

Despite that, there is need of more researches on risk related of COVID-19 to cancer patients to conclude the risk factor. It is essential to report that whether cancer patients in moderate epidemic areas carry a higher risk of COVID-19 infection than the general population in the community. It is already reported that COVID-19 is highly infectious and communicable disease which can affect all the segment of community as well as cancer center. In our study, we shared how our cancer center continued the service to cancer patients during COVID-19 pandemic with all preventive majors. We reported that all the precautions, training, planning, screening and protocols are planned during pandemic at our center i.e. B.P. Koirala Memorial Cancer Hospital Bharatpur, Nepal.

\section{Methods and Strategies Plan}

Strategies for treatment of cancer patients during the COVID-19 pandemic

As the COVID-19 pandemic continues, oncologists must consider the risks of death from COVID-19 versus the large benefits of cancer treatment. However, strategies should be considered to minimize interruptions to cancer care. In the current pandemic environment, patients with cancer are classed as either having confirmed infection or high-risk for COVID-19. It is recommended to establish a multidisciplinary team, including oncologists, respiratory physicians, anesthesiologists, infectious disease physicians, and infection control staff, for the management of patients with cancer and confirmed or suspected COVID-19.

After multiple meeting of different disciplinary expert teams, it was concluded that screening of medical staffs, non-medical staffs, patients and visitors for SARS-CoV-2 should be start. For that either hospital should have own RT-PCR screening laboratory or should sent collected swab sample to any laboratory. At least, hospital should have RT-PCR swab collection facility for screening. Before interning to hospital premises, all the staffs, visitors and patients should go for screening. It was also concluded that whole area of hospital including out patients department (OPD), inpatients department (hospital wards), pathology department, radiology department, administrative department, radiation oncology department and other assessor departments should be sanitized before restart the hospital services for cancer patients. Hospital developed various action plans shown in figure 1-4.

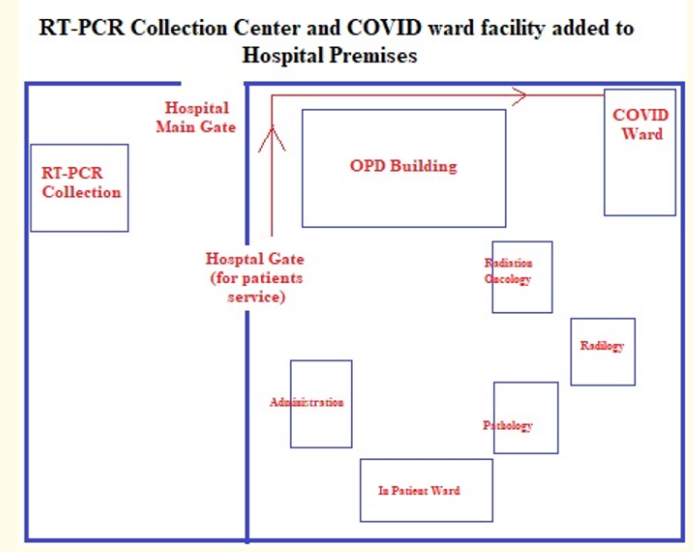

Figure 1: Showing action plan to develop RT-PCR center and Separate COVID Isolation Ward in Cancer Hospital.

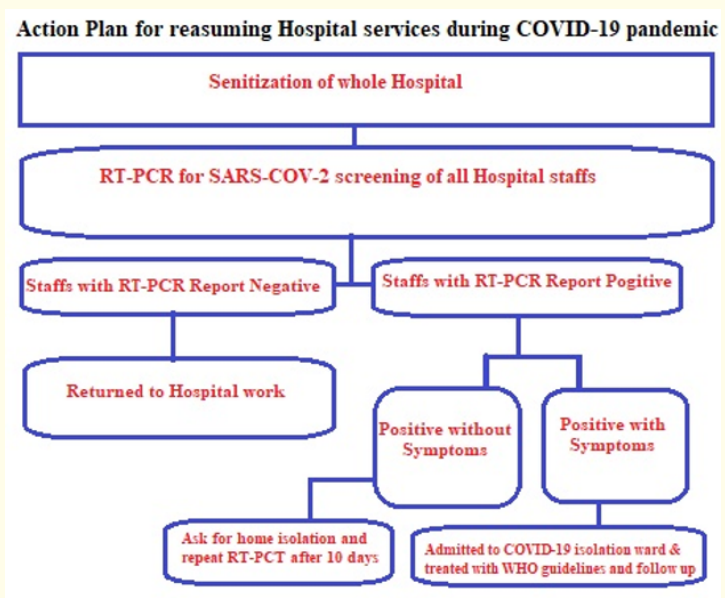

Figure 2: Showing action plan to reassuming hospital services during COVID-19 in Cancer Hospital. 
Action Plan for Resuming OPD and Inpatient Service of Hospital

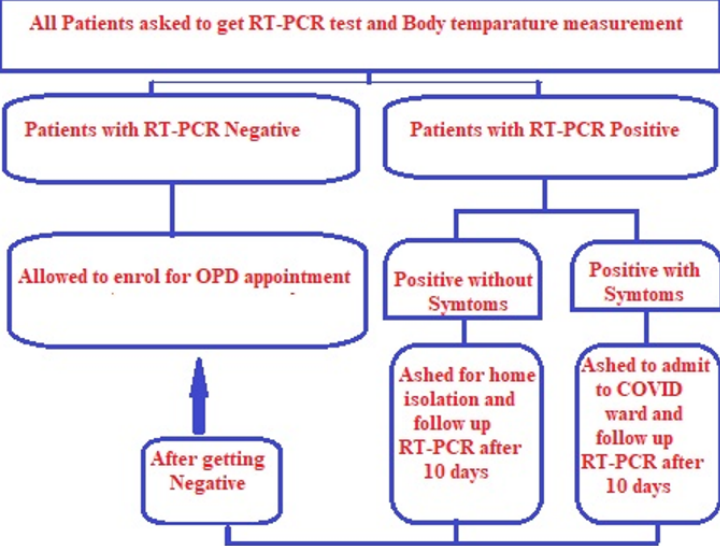

Figure 3: Showing action plan to reassuming all hospital services in cancer hospital.

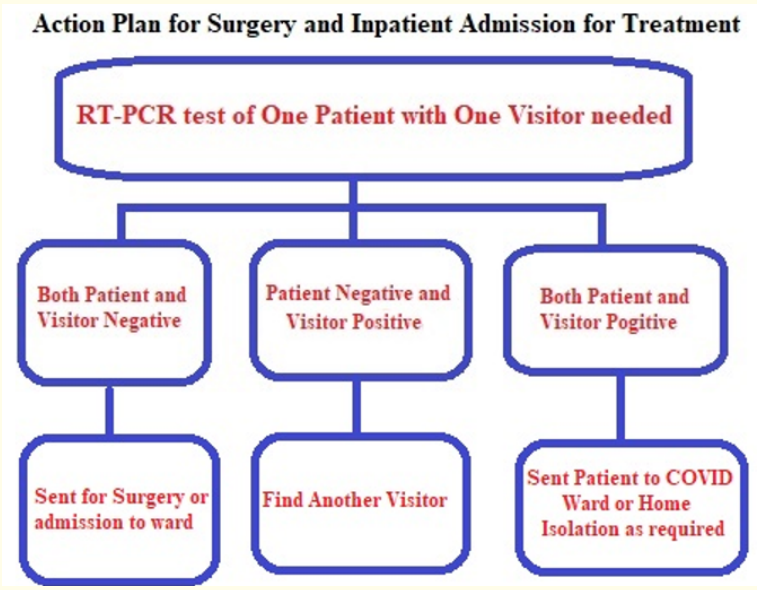

Figure 4: Showing action plan specially for surgery and Inpatient ward facility.

Hospital should start separate isolation COVID-19 Ward for SARS-CoV-2 infected hospital staffs and cancer patients. Hospital should by all protective major equipment like mask, PPE, gloves, Face shield, shielding for hospital staff to minimize the infection rate. There should be other accessory infection control strategies infra-structure development in the hospital like sanitization equipment everywhere in hospital area (Figure 5), hand wash facility with water and soap, availability of sanitizer spray 24 hours, to sanitize throughout hospital area, safety major at diagnostic procedure (Figure 6), wall pomp-lets regarding protection from SARSCoV-2 infection (Figure 7), marking on floor for the social distancing (Figure 8) all those area where people require to be in line like billing (hospital services payment) and miking regarding awareness of COVID-19 prevention.

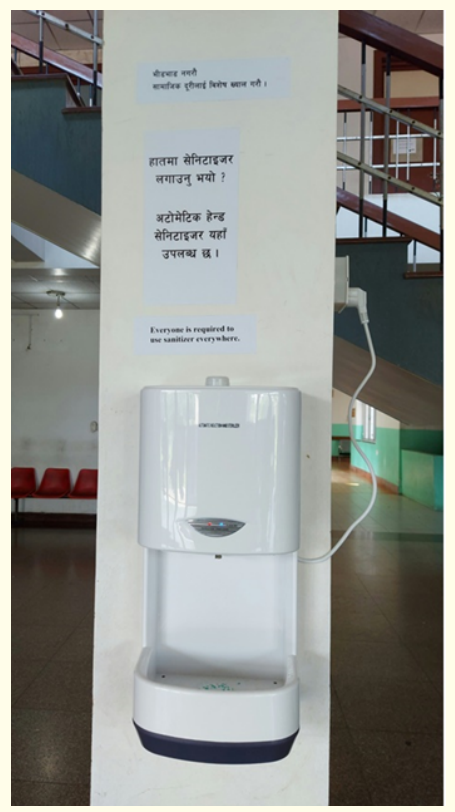

Figure 5: Showing hand sanitizer placed at cancer hospital.

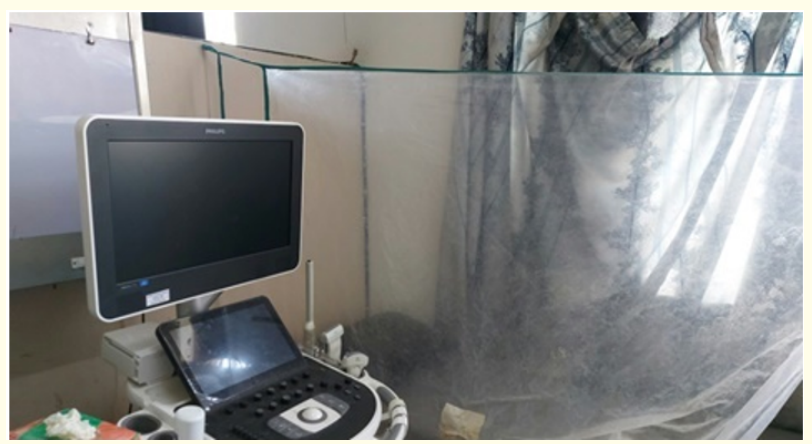

Figure 6: Showing safety major taken at Ultrasonography (USG) in cancer Hospital. 


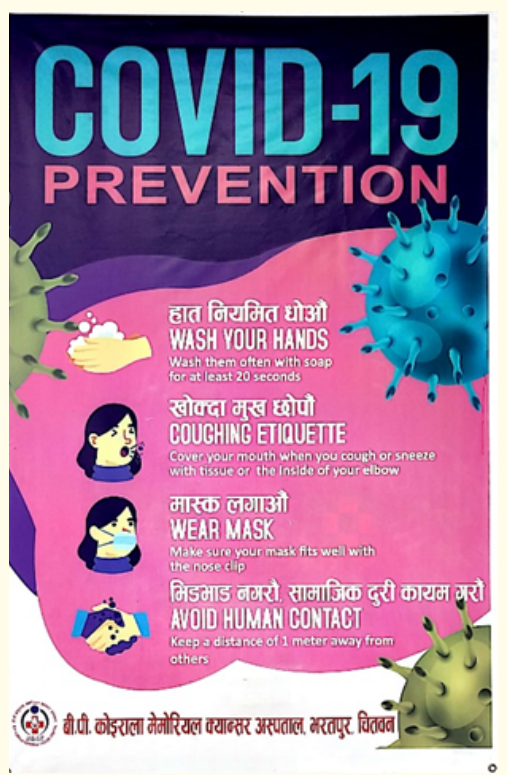

Figure 7: Showing wall pomp-lets regarding protection from SARS-CoV-2 infection, placed throughout all over hospital walls.

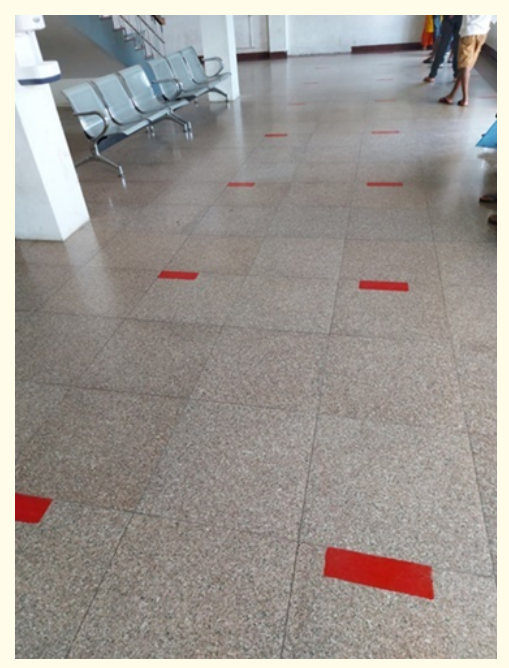

Figure 8: Showing social distancing marking at cancer hospital.

\section{Staff training and different level of protection}

All personnel, including medical professionals and supporting staff, received comprehensive training, virtual/direct discus- sions, meeting and planning on COVID -19 pandemic prevention and control. Different level of protection were provided to our staff with safety equipment like mask, Personal Protective Equipment (PPE) kits, gloves, face shield, shielding. Some other action were taken like adding more sanitization equipment water and soap or sanitizer throughout hospital area, pomp let and miking regarding awareness of COVID-19, Marking on floor for the Social Distancing.

\section{Patient enrollment}

During the epidemic of COVID-19 from March 2020 in our country Nepal, all patient need to schedule an appointment to see the doctor through the hospital contact or through direct contact with their doctor which was provided through social sites. Follow up patients are advised to get phone counseling or online counseling who are unable to visit hospital because of lockdown throughout the Nepal. The patient body temperature was measured upon their arrival at B.P. Koirala Memorial Cancer Hospital. All Patient were advised to wear masks during the visitation in our Hospital. All Individual with fever were sent to fever clinic for travel history investigation, chest examination on CT/X-ray, clinical symptoms assessments such as cough, myalgia, fatigue, and RT-PCR test. Only patients, who were known history of negativeSARS-CoV-2, were enrolled in Out Patient Department (OPD).

Before surgery and ward admission for chemotherapy or radiation therapy or any other interventions, Pharyngeal swab form each patient with one visitor were collected for RT-PCR. Initially Pharyngeal swab were sent to Central COVID Hospital for RT-PCR, later on RT-PCR lab (Quantum 3/5 RT-PCR System, 96-well of Thermo-Scientific Company) was established in our Hospital. Patients with positive RT-PCR test has got admitted to COVID isolation ward or refer to the home isolation depending on patient condition and their cancer treatment will be done after negative RT-PCR test.

\section{RT-PCR test and following up evaluation}

Initially (first week of March 2020), Only RT-PCR swab collection center was installed to our cancer center and all those collected swabs were sent to Central COVID-19 Laboratory, Bharatpur for test. Within few weeks (third week of March 2020), Our hospital established own COVID-19 RT-PCR laboratory with highly specialized and modern equipment i.e. Quantom 3 and 5 Model RT-PCR laboratory equipment with 96-well of Thermo-Scientific Company. All the equipment of RT-PCR COVID-19 laboratory unit at BP Koi- 
rala Memorial Cancer Hospital Bharatpur Nepal are showing in figure 9. The protocol of this Reverse Transcription polymerase chain reaction (RT-PCR) assays targeting the RNA-dependent RNA polymerase (RdRp), nucleocapsid (N) and envelope (E) genes of SARSCoV-2. All three RdRp, $\mathrm{N}$ and $\mathrm{E}$ genes were considered according to COVID-19 Prevention and Control Guideline from National Public Health Laboratory Nepal (10). In our Hospital, RT-PCR Ct value 34 is the cutoff value to distinguish positive and negative amplifications. Patients with all three positive RdRp, Positive $\mathrm{N}$ gene and Positive E genes were reported as SARS-CoV-2 infected patients. If patients tested RT-PCR negative but have all the related symptoms of COVID-19, were sent for further investigation like CT scan and other laboratory blood investigations to confirm it.

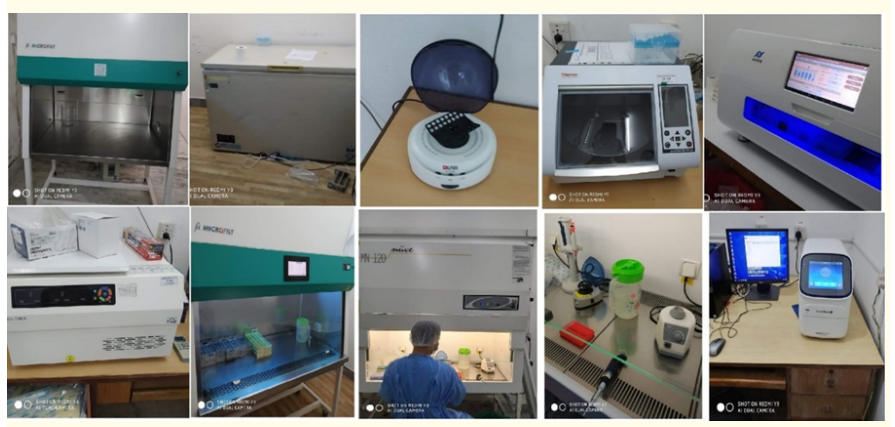

Figure 9: Showing RT-PCR COVID-19 laboratory unit at BP Koirala Memorial Cancer Hospital, Bharatpur, Nepal.

\section{COVID-19 isolation ward}

First week of March 2020, highly equipped and modern COVID Isolation ward (Figure 10) with 20 beds facilities was prepared for SARS-CoV-2 infected hospital staffs and cancer patients. Out of 20 beds, 10 beds are general beds with oxygen supply availability, 5 HDU beds facilities and 5 ICU beds with 2 ventilators availability. When our country badly hit by second wave of SARS-CoV-2 double mutant and triple mutant, there were worse situation in Nepal. Infection transmission rate, mortality rate and daily positivity rate were very high since the last week of April 2021. On the third week of May 2021, our hospital extended COVID isolation ward up to 140 beds from 20 beds and started treating general COVID-19 patients to our hospital.

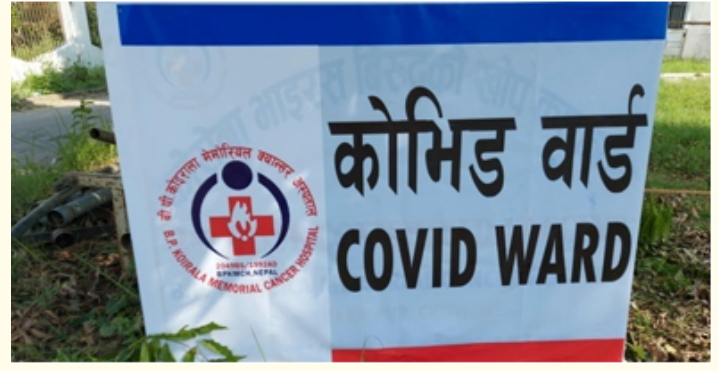

Figure 10: Showing separate Covid Isolation Ward at cancer hospital.

\section{Statistical analysis}

Statistical data and its analysis were provided by hospital medical record department and their team. There is HIS/RIS available at hospital so it was easy to collect medical record.

\section{Results}

On the first screening of all hospital staffs for COVID-19, 16 staffs including medical and non-medical in BP Koirala Memorial Cancer Hospital, Bharatpur were infected by SARS-CoV-2. All these hospital staffs were asymptomatic and sent for home isolation. All these hospital staffs returned back to work after getting negative. According to most updated data provided by medical record department May 2021, 67 hospital staffs got infected by SARS-CoV-2 and recovered from disease too. None of the hospital staff death was reported since the month of May 2021.

A total number of 114273 cancer patients were visited and got Out Patient Department (OPD) Service from March 2020 to May 2021 and Hospital continue providing all patients care service to all patients. According to the clinical basis and demographical basis, all patient information is summarized in table 1 and 2 respectively. All the patients were enrolled for RT-PCR test for SARS-CoV-2and 365 samples were reported positive during this period. None of the cancer patients were reported death due to SARS-CoV-2 infection at hospital during this period.

\begin{tabular}{|c|c|c|c|c|}
\hline Unit & $\begin{array}{c}\text { New } \\
\text { patient }\end{array}$ & $\begin{array}{c}\text { Paid } \\
\text { followup }\end{array}$ & $\begin{array}{c}\text { Free } \\
\text { followup }\end{array}$ & $\begin{array}{c}\text { Total } \\
\text { Patient }\end{array}$ \\
\hline Paediatric & 687 & 2292 & 3308 & 6287 \\
\hline Gynecology & 3076 & 2261 & 2509 & 7846 \\
\hline
\end{tabular}




\begin{tabular}{|c|c|c|c|c|}
\hline $\begin{array}{c}\text { Head and neck } \\
\text { unit }\end{array}$ & 5112 & 3577 & 3462 & 12151 \\
\hline Urology & 936 & 1531 & 1411 & 3878 \\
\hline $\begin{array}{c}\text { Cancer screen- } \\
\text { ing (via) }\end{array}$ & 40 & 0 & 1 & 41 \\
\hline $\begin{array}{l}\text { Nuclear medi- } \\
\text { cine }\end{array}$ & 2 & 2 & 3 & 7 \\
\hline Orthopedic & 1630 & 1108 & 1121 & 3859 \\
\hline $\begin{array}{l}\text { Medical oncol- } \\
\text { ogy } 2\end{array}$ & 1389 & 8727 & 6481 & 16597 \\
\hline $\begin{array}{l}\text { Medical oncol- } \\
\text { ogy } 1\end{array}$ & 2003 & 7090 & 7477 & 16570 \\
\hline $\begin{array}{l}\text { Radiation on- } \\
\text { cology }\end{array}$ & 217 & 11275 & 8438 & 19930 \\
\hline Gi surgery & 3016 & 2234 & 2496 & 7746 \\
\hline Breast surgery & 2716 & 3410 & 4244 & 10370 \\
\hline Neuro surgery & 356 & 213 & 295 & 864 \\
\hline $\begin{array}{c}\text { Stoma care } \\
\text { clinic }\end{array}$ & 4 & 374 & 112 & 490 \\
\hline Physiotherapy & 20 & 1 & 5 & 26 \\
\hline $\begin{array}{l}\text { Urogenital/sar- } \\
\text { coma oncology }\end{array}$ & 1 & 0 & 1 & 2 \\
\hline $\begin{array}{c}\text { Thoracic sur- } \\
\text { gery }\end{array}$ & 1939 & 2621 & 2343 & 6903 \\
\hline Emergency & 696 & 4 & 4 & 704 \\
\hline $\begin{array}{l}\text { Lung and gi } \\
\text { oncology unit }\end{array}$ & 1 & 0 & 1 & 2 \\
\hline Total & 23841 & 46720 & 43712 & 114273 \\
\hline
\end{tabular}

\begin{tabular}{|c|c|c|c|c|c|c|}
\hline 7 & $\begin{array}{c}\text { Province7 } \\
\text { (Surdurpash- } \\
\text { chim) }\end{array}$ & 812 & 749 & 2289 & 3654 & 7504 \\
\hline
\end{tabular}

Table 2: Summary of geographic statistics report (Out patient department \{OPD\} new follow up) (March 2020 to May 2021).

Total numbers of 8012 cancer patients were admitted to ward from March 2020 to May 2021 and 7810 cancer patients discharged from Inpatients ward of the hospital during this period. The detail of all patients who were admitted to ward, are summarized to table 3 .

\begin{tabular}{|c|c|c|c|c|c|c|}
\hline Ward & \multicolumn{3}{|c|}{ Admission } & \multicolumn{3}{c|}{ Discharge } \\
\hline & Male & Female & Total & Male & Female & Total \\
\hline Bed manage & 10 & 11 & 21 & 11 & 12 & 23 \\
\hline Breast surgical unit & 6 & 235 & 241 & 6 & 234 & 240 \\
\hline Cabin & 190 & 195 & 385 & 182 & 186 & 368 \\
\hline Covid-19 ward & 23 & 12 & 35 & 6 & 1 & 7 \\
\hline Gi surgery unit & 156 & 212 & 368 & 153 & 209 & 362 \\
\hline Gynecology & 9 & 320 & 329 & 9 & 315 & 324 \\
\hline Hdu unit & 1 & 0 & 1 & 1 & 0 & 1 \\
\hline Head and neck unit & 344 & 175 & 519 & 331 & 171 & 502 \\
\hline Hematology & 138 & 94 & 232 & 131 & 94 & 225 \\
\hline $\begin{array}{c}\text { Hospice and pallia- } \\
\text { tive care }\end{array}$ & 3 & 3 & 6 & 3 & 1 & 4 \\
\hline Intensive care unit & 1 & 0 & 1 & 1 & 0 & 1 \\
\hline Medical oncology 2 & 210 & 159 & 369 & 199 & 155 & 354 \\
\hline Medical oncology 1 & 603 & 541 & 1144 & 597 & 532 & 1129 \\
\hline $\begin{array}{c}\text { Medical oncology } \\
\text { unit 2 }\end{array}$ & 862 & 698 & 1560 & 847 & 689 & 1536 \\
\hline Neurosurgery & 10 & 11 & 21 & 7 & 11 & 18 \\
\hline Orthopedic unit & 83 & 64 & 147 & 74 & 61 & 135 \\
\hline $\begin{array}{c}\text { Paediatric oncology } \\
\text { unit }\end{array}$ & 233 & 103 & 336 & 230 & 101 & 331 \\
\hline Paediatric oncology & 1 & 0 & 1 & 1 & 0 & 1 \\
\hline Post-operative unit & 126 & 125 & 251 & 130 & 122 & 252 \\
\hline Radiation oncology & 282 & 933 & 1215 & 274 & 924 & 1198 \\
\hline $\begin{array}{c}\text { Thoracic surgery } \\
\text { unit }\end{array}$ & 197 & 257 & 454 & 188 & 246 & 434 \\
\hline $\begin{array}{c}\text { Urogenital/sarcoma } \\
\text { oncology }\end{array}$ & 1 & 0 & 1 & 0 & 0 & 0 \\
\hline Urology unit & 285 & 90 & 375 & 278 & 88 & 366 \\
\hline Anesthesia & 0 & 1 & 1 & 0 & 1 & 1 \\
\hline Biochemistry & 0 & 1 & 1 & 0 & 1 & 1 \\
\hline Emergency & 54 & 31 & 85 & 38 & 27 & 65 \\
\hline Medicine & 1936 & 1597 & 3533 & 1890 & 1568 & 3458 \\
\hline Radiation oncology & 226 & 865 & 1091 & 223 & 853 & 1076 \\
\hline Stomacare clinic & 4 & 4 & 8 & 4 & 4 & 8 \\
\hline Surgery & 1550 & 1736 & 3286 & 1499 & 1695 & 3194 \\
\hline $\begin{array}{c}\text { Thoracic surgery } \\
\text { unit }\end{array}$ & 3 & 7 & 4 & 3 & 7 \\
\hline & 3774 & 4258 & 8012 & 3658 & 4152 & 7810 \\
\hline
\end{tabular}

Table 3: Summary of periodic admission and discharge statistics (March 2020 to May 2021). 
Some of symptomatic and asymptomatic SARS-CoV-2 infected cancer patients who underwent for Computed Tomography for screening. All the symptomatic patients treated at either our hospital COVID ward or Temporary COVID Hospital Bharatpur. None of the cancer patient reported death at our cancer center.

Nevertheless, we encounter with the cancer patient RT-PCR positive without any symptoms (asymptomatic SARS-CoV-2 infected patients) shown in figure 11, at our cancer center on date May, 12 2021. That patient was new patient 34-year-old male with right buccal mucosa carcinoma. That time, patients sent to home isolation and asked to repeat RT-PCR after 14 days and after 14 days, patient was reported RT-PCR negative. On date May 27,2021, patient went through surgery.

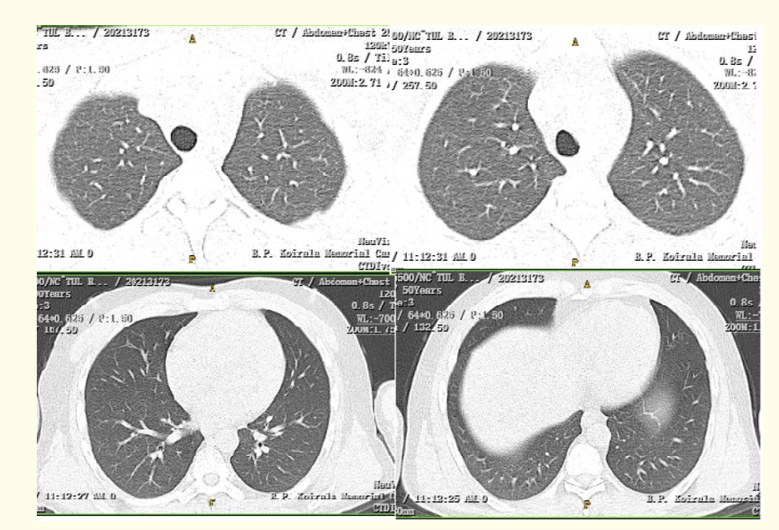

Figure 11: 34-year-old male with right buccal mucosa carcinoma tested RT-PCR positive without any similar symptoms of SARS-

COV-2 virus infection, Chest HRCT was also normal.

We encountered one another 38-year male cancer patient with RT-PCT positive with symptoms (Symptomatic SARS-CoV-2 infected patients) shown on figure 12 and that patient admitted to our COVID-19 ward. After anti-infection and supportive treatment, patient was recovered from COVID-19 and reported RT-PCR negative. One 69-year-oldfemale follow up colon cancer patient (hemicolectomy) who tested RT-PCT negative but had similar symptoms of SARS-COV-2 virus infection. That patient was distinguished as suspectedCOVID-19 patient and sent for chest HRCT. After chest HRCT, Patient reported positive of SARS-COV-2 (Symptomatic SARS-CoV-2 infected patient with false negative RT-PCR) shown in figure 13.

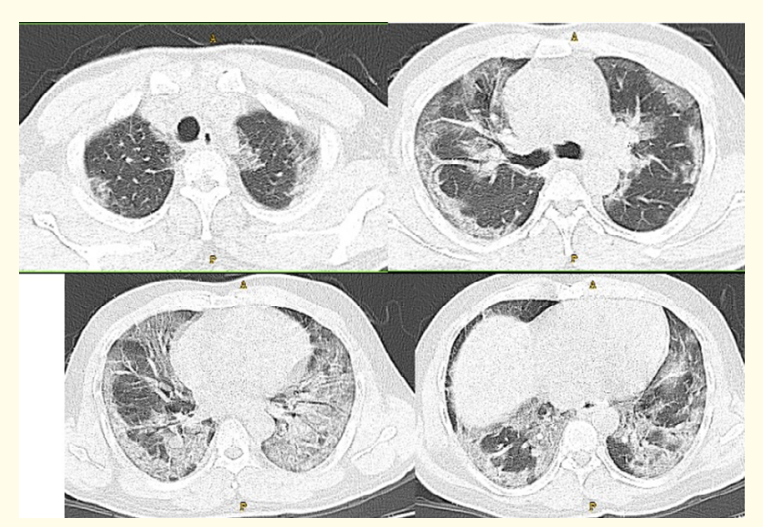

Figure 12: 38-year male cancer patient with RT-PCT positive with symptoms (Symptomatic SARS-CoV-2 infected patients) but recovered after treatment.

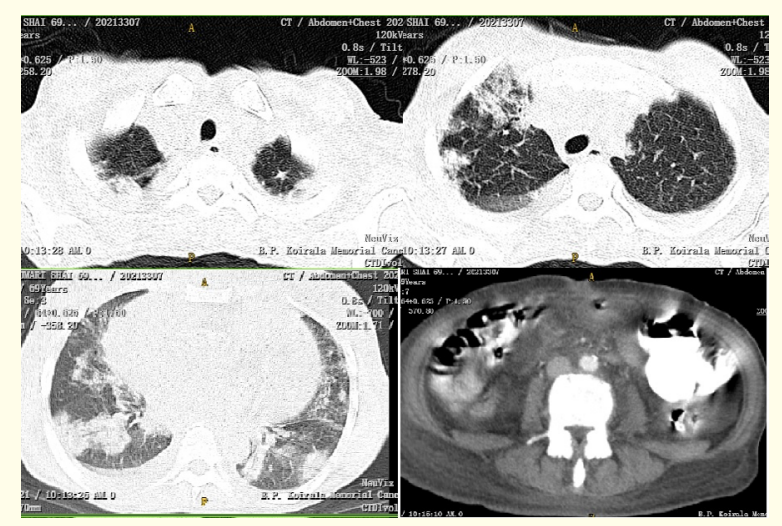

Figure 13: 69-year-old female follow up colon cancer patient (hemicolectomy), tested RT-PCT negative but had similar symptoms of SARS-COV-2 virus infection. After chest HRCT, Patient reported positive of SARS-COV-2 (Symptomatic SARS-CoV-2 infected patient with false negative RT-PCR).

\section{Discussion}

The strategies plans prepared by hospital expert team were executed at cancer center Bharatpur and all the hospital services reassumed. Our hospital is still proving timely oncology services to a large number of cancer patients as well as from May 2021 providing service to general COVID-19 patients by the order of Gov- 
ernment of Nepal. Although, there were a few number of hospital staffs got infected with SARS-COV-2 virus but all of them recovered soon. A few number of cancer patients were also found positive with SARS-COV-2 virus and all of them recovered and treatment of cancer continued after recovery. The action plan prepared by our expert team became successful and a large number of cancer patients got required medical services. All the services are still continuing in our cancer center. Some of our hospital staffs got infected but that might be from market during buying food stuffs or any other reasons. There is also chance of false negative report of patient due to error or various other reasons. It was reported that findings of RT-PCR emphasize the need for repeated testing in patients with suspicion of SARS-Cov-2 infection symptoms given that up to $54 \%$ of COVID-19 patients may have an initial false-negative RT-PCR (very low certainty of evidence) [11]. On the other hand, other cancer center recommended examining carcinoma patients with only active therapy [12], it could decrease the sample size and work load to clinical laboratory. But our strategies plan suggested additional preclusion technique must be firmly used to prevent inhospital transmission by OPD patients and patients' companions, especially in region with a high incidence of SARS-CoV-2 infection.

It is reported that there are certain clinical symptoms between SARS-CoV-2 infected patients and cancer patients, including fever and low leukocyte count which caused by carcinoma or chemotherapy. Oncologist can distinguish the progression of fever and lung infection with the help of lung CT images $[6,13,14]$. However, it is very difficult to distinguish pneumonia due to SARS-CoV-2 infection from aspiration pneumonia and radiation induced pneumonitis. In our cancer center, RT-PCR test for SARS-CoV-2 infection are theoretically supposed gold standard of COVID-19 case finding. Patients with positive HRCT chest with other clinical symptoms were reported as clinically COVID-19 cases.

A clinical study reported five patients who diagnosed as SARSCoV-2 infected patients but their RT-PCR report were negative. That study concluded that insufficient viral samples and laboratory problems might be accountable for the false-negative rate of SARSCoV-2 RT-PCR testing [15]. One research concluded that positive rate of sputum sample has higher than nasal or pharyngeal swabs [16]. Most of the patients don't have wet cough and sputum sample can't be collected. At our cancer center, only nasal and pharyngeal sample were collected for RT-PCR COVID-19 test. One clinical study also suggested that those patients who have no sputum, pharyn- geal swab could still be used for testing because SARS-CoV-2can be found in saliva samples [17].

Patients with carcinoma already have weakened immunity system, after treatment procedures like chemotherapy and radiation therapy which additionally caused decrease in immunity of patients, are very prone to pneumonia. During swine flu (H1N1 influenza) pandemic in 2009, it was reported that ICU admission rate and mortality rate for patients with hematologic malignancies and/or hematopoietic stem cell transplant were $31 \%$ and $22 \%$ respectively [18]. Even for the healthy people, it was reported that ICU admission rate and mortality rate were $25 \%$ and $7 \%$ respectively during swine flu (H1N1 influenza) pandemic in 2009 [19].

During the RT-PCR screening of SARS-COV-2 virus in cancer patients at BP Koirala Memorial Cancer Hospital Bharatpur in Nepal, we found that most of the positive diagnosed cases were asymptomatic and after 10 - 14 days home isolation, they became negative. A few numbers of infected patients have symptoms like fever and dry cough and after screening with chest HRCT, there were diagnosed as pneumonia. After treatment in COVID-19 isolation ward of our hospital, they were recovered. Hospital staffs that were infected with SARS COV-2 during month of March, April 2020, all were asymptomatic. But during second wave of COVID-19 in the month of May 2021, a few numbers of staffs that were infected with SARS COV-2 virus have minor symptoms like fever. After screening with chest HRCT, none of them are reported with chest infection or pneumonia.

During the epidemic of SARS COV-2 virus, several scientific associations developed the guidelines regarding cancer patient care or management of cancer patient treatment during COVID-19 pandemic. The Italian Association of Medical Oncology (AIOM) recommended that assess case by case for probability of delaying a treatment, considering the biological characteristic of cancer, the clinical aspects of the patient and the potential health threats for SARS COV-2 virus infection [20]. On the other hand, French oncology guidelines suggest that treatment management of patients with carcinoma during epidemic of SARS COV-2 virus infection, should be depend on the criteria like curative, non-curative, age, life expectancy, time since diagnosis and symptoms [21]. The National Institute of Health and Care Excellence (NICE) developed a guideline for telephone or online consultation for follow up patients and to minimize the face to face contact and home delivery of medicine to 
door to door and even for blood tests [22]. Whereas European Society of Medical Oncology (ESMO) developed a guideline for cancer patients during COVID-19 pandemic according to priority based i.e. who needed most [23]. The American Society of Clinical Oncology (ASMO) guidelines focused on delivery maximum information to patients regarding health care during COVID-19 pandemic, prevention guidelines developed by American Center for Disease Control (CDC) regarding SARS COV-12 virus [24,25]. But our hospital expert team recommended that provide services to all cancer patients with screening them RT-PCR test SARS-COV-2 and differentiate infected and non-infected patients first. After differentiate the cancer patients either infected or not-infected with SARS-COV-2 virus, allowed non-infected to get all required services from hospital. All infected cancer patients with SARS-COV- virus sent to either home isolation or COVID isolation ward according to symptoms and clinical condition of patients. After recovery of infected cancer patients from SARS-COV-2 virus, they were also allowed to get all hospital treatment facility related to carcinoma diseases. This is an only local guideline which was adopted by our cancer hospital. We also used all protective equipment like personnel protecting equipment (PPE), gloves, protective goggles, face shield, face mask and all protecting guidelines like social distancing, hand wash frequently, frequently sanitize hand, sanitization of infected building and area. Although, our hospital staffs were infected by SARS-COV-2 virus, what I think, it may be from outside when buying food stuffs or any other activities. But none of our hospital staff gets any serious problem and recovered easily from COVID-19. On date January 29,2021 , all hospital staffs got first dose of Covishield vaccine and second dose of vaccine was given to all hospital staff on April 20, 2021. At the time of preparing this article, there is strict lockdown in district named Chitwan where our cancer hospital. In spite of strict lockdown, our cancer center is running with full capacity and providing all services to cancer patients. Not only providing hospital services to cancer patients with same strategies developed by local experts but also hospital developed 140 beds COVID isolation ward with all modern equipment and providing treatment facilities to general COVID-19 patients. We suggest that it is better and can also minimize the transmission of diseases.

Although the treatment of cancer patients diagnosed with SARS-COV- virus is difficult. But local guidelines for COVID-19 prevention and control recommended that all patients, cancer patients included, with confirmed infections, must be transferred to designated hospitals with multidisciplinary medical teams that one is developed at our cancer center. According to the clinical situation of cancer patients, treatment procedure was chosen on the basis of benefit verses adverse effect such as immunosuppression in patient's immune system. Cancer related treatment is also immunosuppressive like chemotherapy and radiation therapy so it is recommended that delaying in cancer related treatment until the patient got recovered from COVID-19. Initially, anti-infection therapy and symptomatic treatment was given to infected patients. During the infection control therapy, those symptoms which might be develop with cancer patients like airway obstruction, internal bleeding must be monitored and treated. There are a lots of local guidelines developed by different cancer hospital and have adopted to treat infected cancer patients [26-28].

\section{Conclusion}

Thanks to our expert team who developed strategies plan and executed that strictly to provide hospital services all the infected and non-infected cancer patients with SARS-COV-2 separately by developing a separate COVID-19 isolation ward. Our study suggested that all-inclusive set of SARS-COV-2 virus precautionary intrusions joint with RT-PCR test for SARS-CoV-2 (nucleic acid test) could successfully implemented and accomplish cancer patients, and eventually promote patient care. We also concluded that cancer patients infected with COVID-19 of Nepal are not at higher risk than general COVID-19 infected patients. Our study strongly suggested that adding RT-PCR screening facility and separate COVID isolation ward including all preventive majors regarding COVID-19 to our hospital, makes more impact on cancer patients treatment management during COVID-19 pandemic.

\section{Bibliography}

1. "Coronavirus resource center". Jons Hopkins University of Medicine (2021).

2. “COVID-19 Symptoms, Coronavirus Disease”. World Health Organization (WHO).

3. Symptoms, COVID-19, Centers for Disease Control and Prevention (CDC).

4. Jingjing He., et al. "Proportion of asymptomatic coronavirus disease 2019: A systematic review and meta-analysis". Journal of Medical Virology 93.2 (2021): 820-830. 
5. “Covid-19 update portal". Ministry of Health and population, Government of Nepal (2021).

6. Chen N., et al. "Epidemiological and clinical characteristics of 99 cases of 2019 novel coronavirus pneumonia in Wuhan, China: a descriptive study". Lancet 395.10223 (2020): 507-513.

7. Kamboj M and Sepkowitz KA. "Nosocomial infections in patients with cancer". Lancet Oncology 10.6 (2009): 589-597.

8. Jianbo Tian., et al. "Clinical characteristics and risk factors associated with COVID-19 disease severity in patients with cancer in Wuhan, China: a multicentre, retrospective, cohort study". Lancet Oncology 21.7 (2020): 893-903.

9. Xia Y., et al. "Risk of COVID-19 for patients with cancer". Lancet Oncology 21.4 (2020): e180.

10. National Testing Guidelines for COVID-19 (Approved Date: 2077/02/20| Approved by Hon. Minister, Ministry of Health and Population Nepal).

11. Ingrid Arevalo-Rodriguez., et al. "False-negative results of initial RT-PCR assays for COVID-19: A systematic review". PLoS One 15.12 (2020): e0242958.

12. Lu R., et al. "Genomic characterisation and epidemiology of 2019 novel coronavirus: implications for virus origins and receptor binding". Lancet 395.10224 (2020): 565-574.

13. Wang D., et al. "Clinical Characteristics of 138 Hospitalized Patients With 2019 Novel Coronavirus-Infected Pneumonia in Wuhan, China". JAMA 323.11 (2020): 1061-1069.

14. Chua F., et al. "The role of CT in case ascertainment and management of COVID-19 pneumonia in the UK: insights from high-incidence regions". Lancet Respiratory Medicine 8.5 (2020): 438-440.

15. Xie X., et al. "Chest CT for Typical Coronavirus Disease 2019 (COVID-19) Pneumonia: Relationship to Negative RTPCR Testing". Radiology 296.2 (2020): E41-45.

16. Wang W., et al. "Detection of SARS-CoV-2 in Different Types of Clinical Specimens". JAMA 323.18 (2020): 1843-1844.

17. Wolfel R., et al. "Virological assessment of hospitalized patients with COVID-2019”. Nature 581.7809 (2020): 465-469.

18. Tramontana AR., et al. "Oseltamivir resistance in adult oncology and hematology patients infected with pandemic (H1N1) 2009 virus, Australia". Emerging Infectious Diseases 16.7 (2010): 1068-1075.
19. Jain S., et al. "Hospitalized patients with 2009 H1N1 influenza in the United States, April-June 2009". The New England Journal of Medicine 361.20 (2009): 1935-1944.

20. Rischioinfettivo da coronavirus COVID 19: indicazioni per l'oncologia (2020).

21. You B., et al. "The official French guidelines to protect patients with cancer against SARS-CoV-2 infection". Lancet Oncology (2020): S1470-2045 (20): 30204-7.

22. "COVID-19 rapid guideline: delivery of systemic anticancer treatments". NICE guideline (2020).

23. "ESMO Guidelines: Cancer patient management during the COVID-19 pandemic" (2020).

24. “COVID-19 patient care information" (2020).

25. “CDC guidance for COVID-19” (2020).

26. Tagliamento M., et al. "Call for ensuring cancer care continuity during COVID-19 pandemic”. ESMO Open 5.3 (2020): e000783.

27. Zhang L., et al. "Clinical characteristics of COVID-19-infected cancer patients: a retrospective case study in three hospitals within Wuhan, China". Annals of Oncology 31.7 (2020): 894901.

28. Lambertini M., et al. "Cancer care during the spread of coronavirus disease 2019 (COVID-19) in Italy: young oncologists' perspective". ESMO Open 5.2 (2020): e000759.

\section{Volume 5 Issue 8 August 2021}

\section{(C) All rights are reserved by Yadav Ajay Kumar., et al.}

